\title{
Sand Blocking Mechanisms and Productivity Analysis of Slant Well in Siltstone Reservoirs
}

\author{
Peifeng Jia $\mathbb{D}^{1,2}$ Chuanzhi Cui $\mathbb{D}^{1},{ }^{1}$ Yizhong Zhao, ${ }^{2}$ Xiukun Wang $\mathbb{D}^{3}$, and Yingfei Sui $\mathbb{D}^{1}$ \\ ${ }^{1}$ College of Petroleum Engineering, China University of Petroleum, Qingdao, China 266580 \\ ${ }^{2}$ Petroleum Engineering Institute of Shengli Oil Field, SINOPEC, Dongying, China 257015 \\ ${ }^{3}$ Unconventional Petroleum Institute, China University of Petroleum, Beijing, China 102249
}

Correspondence should be addressed to Chuanzhi Cui; ccz2008@126.com

Received 27 October 2020; Revised 15 December 2020; Accepted 10 May 2021; Published 27 May 2021

Academic Editor: Guanglong Sheng

Copyright () 2021 Peifeng Jia et al. This is an open access article distributed under the Creative Commons Attribution License, which permits unrestricted use, distribution, and reproduction in any medium, provided the original work is properly cited.

The siltstone reservoir with many small layers of pay zones is usually produced by slant holes. However, severe sand blockages take place when the siltstone reservoir is developed by slant holes. Currently, the sand blocking mechanisms and the effect of sand blocking on productivity of slant well are still challenges for engineers and scholars. In this paper, based on the existing productivity model of inclined slant wells, the mechanical skin factor, which describes the effect of sand blocking on productivity, is proposed. Meanwhile, many experimental works have been done to investigate the sand blocking mechanisms in siltstone reservoirs. From the experimental work, it is concluded that with the increase of displacement PV number and displacement flow rate, the permeability of sand control system decreases by $40 \%$. When solid particles enter the casing and block the gravel and sand control screen in the annulus, the skin factor increases sharply and the productivity decreases by more than $80 \%$. Through the productivity calculation of multilayer sand control wells, it is considered that larger gravel packing radius can keep particles away from the well bore, which is helpful to ensure oil well productivity. Furthermore, the influence of differential filling radius on the fluid production capacity of each layer of sand control well is analyzed. It is proved that optimizing the filling radius of each layer can improve the production effect of mediate- and low-permeability layers. This method has been applied in Kendong \#12 block. The daily oil production rate is increased by $9.61 \mathrm{t} /$ day, and the oil recovery of this block is increased by $2.12 \%$.

\section{Introduction}

The fine siltstone reservoir framework has small grain size and high argillaceous content, and the migration of reservoir particles intensifies with the increase of water cut. This kind of reservoir shows continuous sand production in high water cut period. In production, the oil well skin factor increases, the screen pipe and filling layer are blocked, and the liquid production and flowing pressure decrease [1-7]. The sand production commonly leads to the increase of skin factors, the blockage of the screening tube, and the decrease of both fluid rates and bottom-hole flowing pressure [8-10]. Moreover, the frequent plugging removal operations cost additional maintenance investment and occupy the normal production time [11]. Therefore, effective productivity pre- diction methods of this type of sand-producing wells are needed to guide the optimization of sand control parameters for oil wells and improve the sand control strategies.

Currently, the existing production analysis methods mainly include production profile testing, physical simulating experiments, formation coefficient splitting method, theoretical analytical formulas, and numerical simulations [1219]. These methods mainly use the original geological interpreted formation data to predict the productivity of a well, which lacks a detailed description of the sand control tube and cannot dynamically predict the productivity loss caused by sand blockage. Therefore, in this work, a study on the clogging law of the near-well sand-controlling system was carried out, and the productivity model of sand-producing wells was improved and utilized to optimize the sand control 
parameters. Meanwhile, the sand control strategies were developed. The methodology was validated by the field practice in a typical siltstone reservoir block of Shengli oilfield.

\section{Blockage Mechanisms of Sand Control System in Siltstone Reservoirs}

2.1. Sand-Controlling Slant Well and Its Productivity Model. The typical siltstone reservoir has many discontinuous lateral small pay zones. Multiple layers are usually produced together in inclined vertical wells with sand control treatment for every layer. Generally, the Cinco-Ley productivity model is used in multilayer combined production wells, as shown in equation (1). The flow process of the sand control well is described as a plane radial flow problem in a bounded heterogeneous formation, where each small layer is individually supplied with liquid driven by the production pressure drop. What describes the impact of sand control technology on productivity is the mechanical sand control skin coefficient $S_{d}$. This coefficient is obtained by the well test method, and it is a static parameter. It is impossible to dynamically analyze the effect of blockage on production in the productivity calculation.

$$
\left\{\begin{array}{l}
Q=\sum_{i=1}^{n} Q_{i}, \\
\Delta P_{i}=P_{H}-P_{i}, \\
Q_{i}=\frac{0.54287 k_{i} h_{i} \Delta p_{i} /(\mu B)}{\ln \left(R_{e} / R_{w}\right)+S_{\theta}+S_{d}},
\end{array}\right.
$$

where $Q$ is the production rate of the production well $\left(\mathrm{m}^{3} /\right.$ day $), Q_{i}$ is the production rate of the $i$-th layer of pay zone ( $\mathrm{m}^{3} /$ day), $k_{i}$ is the permeability of the small layer $\left(10^{-}\right.$ $\left.{ }^{3} \mu \mathrm{m}^{2}\right), h_{i}$ is the effective thickness of the layer (m), $\mu$ is the reservoir fluid viscosity (mPa.s), $B$ is the volume coefficient of the reservoir fluid, $R_{e}$ is the outer boundary radius $(\mathrm{m})$, $R_{w}$ is the drainage radius $(\mathrm{m}), S_{\theta}$ is the well deviation and perforation skin coefficient, and $S_{d}$ is the mechanical sand control skin coefficient.

In order to analyze the impact of sand control layer blockage on oil well productivity, as shown in Figure 1, the near-well flow is refined into four parts: screen tube, annulus packing, squeeze packing, and formation reservoir. The boundaries are the sand control screen radius $R_{w}$, the casing radius $R_{t}$, the extrusion filling radius $R_{j}$, and the supply radius $R_{e}$, respectively. The corresponding permeability is screen pseudopermeability $k_{s}$, annular packing permeability $k_{\text {sand }}$, squeezed packing permeability $k_{j}$, and reservoir permeability $k$. The impact of sand control on oil well productivity is mainly described by the mechanical sand control skin coefficient $S_{d}$, as shown in

$$
S_{d}=\frac{k}{k_{j}} \ln \frac{R_{e}}{R_{j}}+\frac{k}{k_{\text {sand }}} \ln \frac{R_{j}}{R_{t}}+\frac{k}{k_{s}} \ln \frac{R_{t}}{R_{w}}-\ln \frac{R_{e}}{R_{w}} .
$$

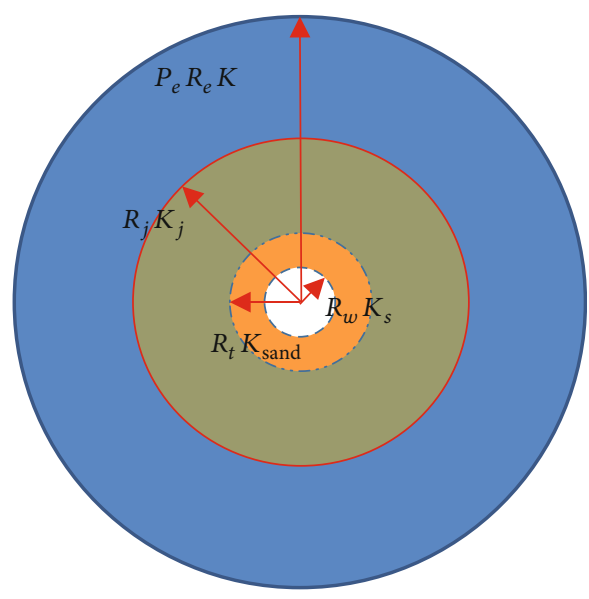

FiguRE 1: Schematic of the near-well scenarios of a sand control well.

$R_{w}, R_{t}, R_{j}$, and $R_{e}$ are screen radius, casing radius, squeezed packing radius, and oil drainage radius, respectively $(\mathrm{m}) ; k_{s}, k_{\text {sand }}$, and $k_{j}$ are screen pseudopermeability, annulus packing permeability, and extrusion permeability of pressure packing layer, respectively $\left(10^{-3} \mu \mathrm{m}^{2}\right)$.

The initial values of parameters required to calculate the skin coefficient of mechanical sand control are given in the design of sand control wells. In the field production process, formation particles continue to migrate to the wellbore, and the permeability of each part of the sand control system is blocked to a certain extent. It is necessary to fully consider the change of sand control skin coefficient caused by blockage in the production capacity prediction process.

2.2. Blocking Mechanism of Sand Control System outside Casing. The main production characteristics of siltstone oilfields in high water cut period are high displacement and high liquid content [20]. The sand production of the reservoir is mainly manifested as continuous sand production leading to blockage of near wells, and violent sand production is rare. The decline in oil well productivity is mainly caused by the blockage of the formations, screens, and blast holes near the well. Although pickling and other technological measures can remove the blockage of the screen and blast hole, they cannot completely eliminate the blockage of the formation near the well [21]. During the experiment, the ring pressure of the displacement pipe was $8 \mathrm{MPa}$, which was close to the pressure of conventional sandstone reservoirs. Since the target reservoir is conventional thin oil, the flow process is less affected by temperature, so the experiment was carried out under normal temperature conditions.

Manufacture the displacement device of the target block formation sand and the packed gravel in series, and carry out the displacement experiment of the packing layer to analyze the relationship between displacement, flow rate, and permeability. The experimental setup is shown in Figure 2. The area where the formation sand and the filling sand are mixed is defined as the sand-mixed layer. Simulation of formation sand is the sand washed out from oil wells in typical blocks, 


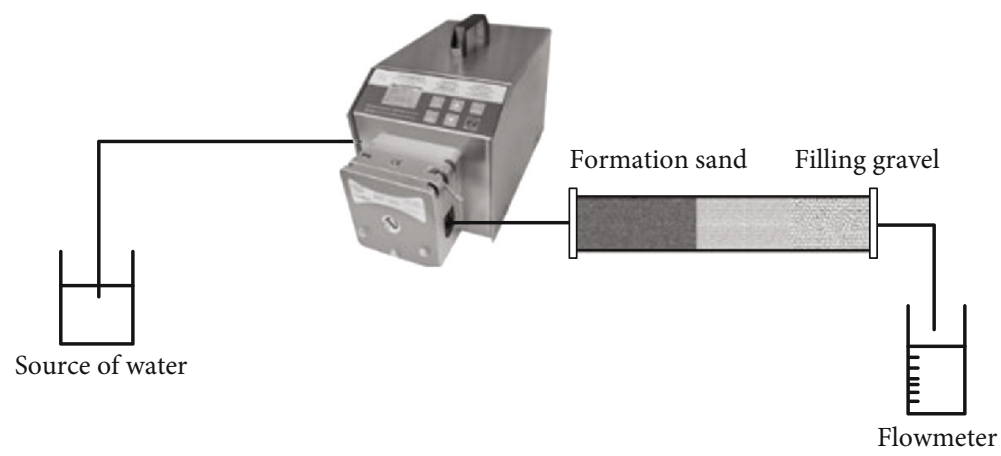

FIGURE 2: Sand-mixed displacement experiment device.

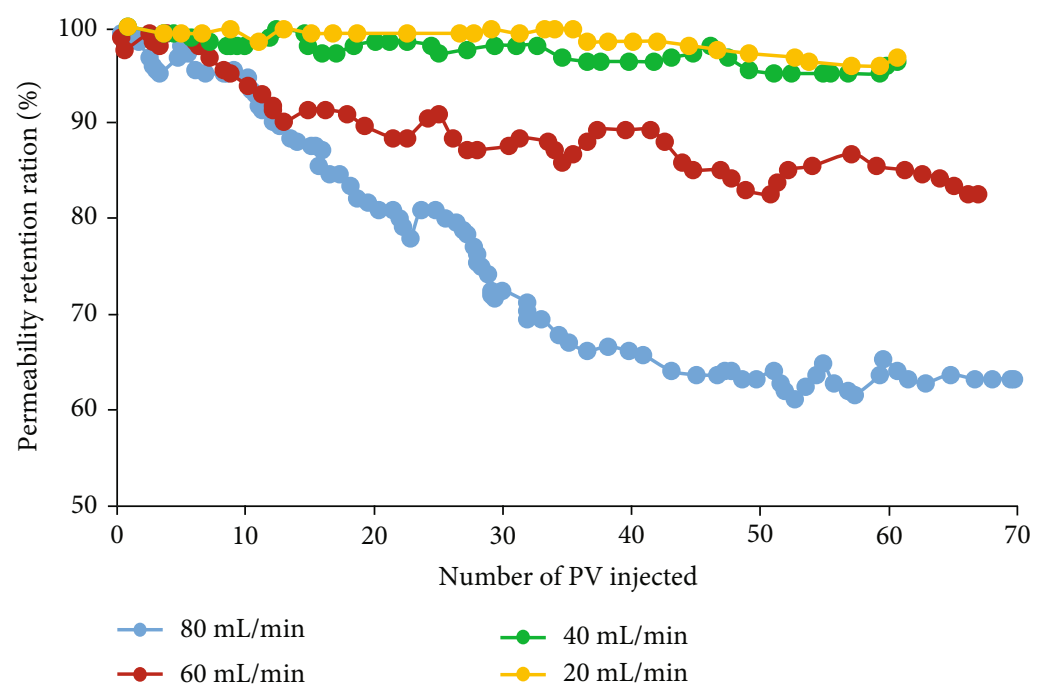

Figure 3: Permeability blockage of the filling layer at different flow rates.

which can well reflect the framework and migration particles of siltstone. Simulation of filling sand is $0.45-0.85 \mathrm{~mm}$ gravel commonly used in a production site. Displacement fluid is brine with a certain amount of thickener. One end of the displacement device is connected with the atmosphere and the other end is equipped with a pressure gauge.

By continuously measuring the outlet flow rate and displacement pressure difference, we can further calculate the dynamic permeability of porous media composed of mixed sand. After flooding with different PV numbers, the permeability of the sand-mixed layer is measured in stages, and the test results are shown in Figure 3.

During the clogging process, the location of the interface between the sand-mixed layer and the simulated formation was blocked first, and then, the blocked position extended to both ends, and the permeability of the sand-mixed layer gradually decreased and finally stabilized. Analyzing the changes in the permeability of the displacement core under different flow rates, it can be seen that as the displacement PV number increases, the permeability gradually decreases. When the flow rate is low, the core permeability is better preserved. As the flow velocity increases, the permeability decreases significantly. With a displacement of $80 \mathrm{~mL} / \mathrm{min}$, the core permeability remains only $60 \%$ after the displacement is stable, which means that near-well blockage will increase under high fluid production intensity. By fitting the chart, the permeability decrease rule of the filling layer of the target block due to blockage can be obtained, as shown in formula (3), where $q$ represents the daily fluid production of the target layer after production, $V_{\Phi}$ represents the void volume of the formation in the sand control system, and $t$ represents the production time. This formula is used as an experimental rule of filling layer blockage in the production capacity prediction.

$$
k_{f}=f\left(q, t, V_{\phi}\right) \text {. }
$$

2.3. Blocking Law of Sand Control System in Casing. After the squeeze filling of the casing perforation completion of the inclined vertical well is completed, special tools are used to fill the annulus of the screen with gravel to reduce the impact on the screen at the blast hole [17]. After formation, solid particles broke through the sand control system outside the pipe and flowed into the wellbore, and the sand control system in the casing composed of "annular gravel screen" began to block. In order to analyze the impact of the blockage of the sand control system in the casing on the productivity, a full-scale "annular gravel screen" permeability test equipment was designed, as shown in Figure 4. The full-size screen 


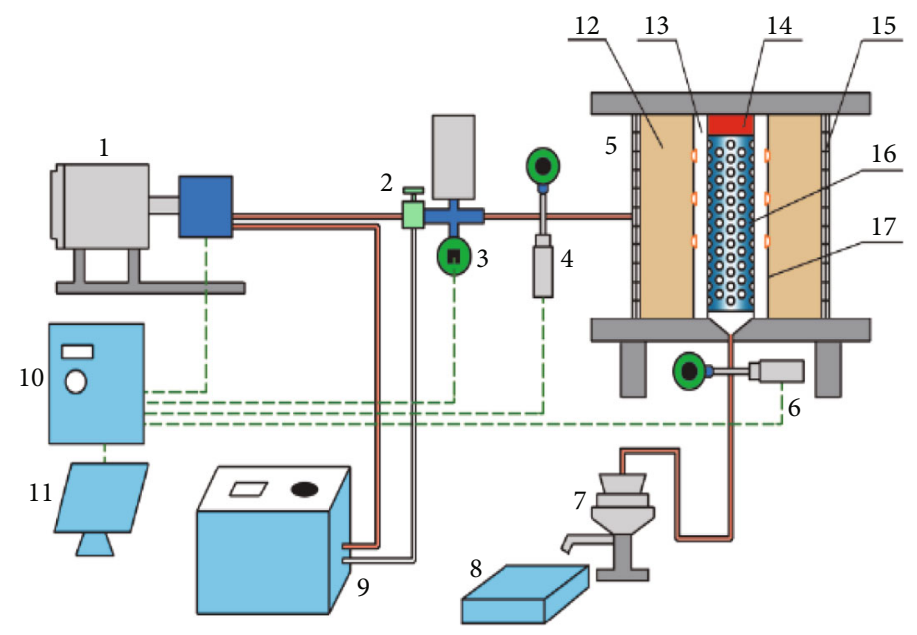

Figure 4: Permeability testing equipment for sand control system in casing. 1-diaphragm pump, 2-safety valve, 3-flow meter, 4-inlet pressure sensor, 5-simulated wellbore, 6-outlet pressure sensor, 7-vibrating screen, 8-collection tank, 9-sand mixing system, 10-servo system, 11-computer data acquisition system, 12-stratum sand, 13-annular space, 14-sealing rubber gasket, 15-diversion net, 16-screen, and 17-support frame.

is placed in a steel cylinder with holes, and the outside of the cylinder is filled with gravel to simulate the sand control well. The formation sand and brine are mixed in the mixing device and flow to the wellbore through the servo system. The data of pressure, flow, and sediment concentration are obtained by sensors and transmitted to the computer.

In the experiment, stratum sand with a median diameter of $0.2 \mathrm{~mm}$ and a homogeneity coefficient of 1.8 was used as the sand source. Two specifications are selected for gravel packing, namely, $0.3-0.6 \mathrm{~mm}$ quartz sand and $0.4-0.8 \mathrm{~mm}$ quartz sand. A short section of a plasma slotted screen with a sand retaining accuracy of $0.15 \mathrm{~mm}$ is selected for testing, and the screen has a pseudopermeability of $142000 \times 10^{-3} \mu$ $\mathrm{m}^{2}$. The displacement experiment can obtain the displacement fluid volume $V_{1}$, the screen-annular gravel pore volume $V_{\mathrm{s}}$ is a fixed value, and the experimental displacement $\mathrm{PV}$ number is defined as the ratio of $V_{1}$ to $V_{s}$. Through experiments, the permeability of the sand control system in the pipe can be measured in real time, and the pseudopermeability $k_{s}$ of the screen pipe and the permeability $k_{\text {sand }}$ of the gravel packing in the annulus can be obtained according to

$$
\left\{\begin{array}{l}
k_{c}=\frac{0.06 q_{c} \mu_{c}}{2 \pi h \Delta P_{c}} \ln \frac{r_{\text {out }}}{r_{\text {in }}}, \\
k_{s}=\frac{k_{c} k_{\text {sand }} \ln \left(r_{s} / r_{\text {in }}\right)}{k_{\text {sand }} \ln \left(r_{\text {out }} / r_{\text {in }}\right)-k_{c} \ln \left(r_{\text {out }} / r_{s}\right)} .
\end{array}\right.
$$

As shown in Figure 5, due to the small filling volume of the near-well annulus, the displacement PV number does not exceed 15 when the permeability of the sand control system is stable. This also shows that after the sand control well is put into production, the blockage near the casing will form a balance in a short time, the blockage permeability remains only $20 \%$, and the production is manifested as a sudden drop in production. At this time, the sand control system is considered to have failed. Comparing the plugging conditions of the packed gravel of different particle size grades, it can be found that for the silty sandstone reservoirs whose particle diameter is generally below $0.25 \mathrm{~mm}$, using $0.4-0.8 \mathrm{~mm}$ gravel packing, the permeability retention is better. Through the observation process, it can be found that the smaller solid particles in the early stage of displacement can flow out through the sand control system, which allows the permeability of the larger-sized packed gravel to be retained. As the displacement progresses, the final permeability retention rates of the two types of gravels are close. By fitting this chart, we can get the law of permeability drop caused by blockage of the sand control system in the pipe, as shown in

$$
k_{c}=f\left(Q_{i}, t, V_{\phi}\right) \text {. }
$$

\section{Productivity Analysis and Application of Multilayer Combined Sand Control Wells in Siltstone Reservoirs}

3.1. Productivity Prediction of Slicing Sand Control Inclined Vertical Well. Based on the revised productivity model of layered sand control wells and the blockage law of sand control system, the productivity of multilayer combined sand control wells in siltstone reservoirs can be predicted. The grid model of near wellbore coordinate is established by using numerical analysis software. The scale of the model is limited to the formation within $50 \mathrm{~m}$ around the well. Kriging method is used to interpolate the stratum attribute model. The finite difference method is used in the solving process, and the specific solving steps are shown in Figure 6. After inputting the geological, technological, and production parameters related to productivity, variables such as the fluid production of each layer, the permeability of the squeeze filling layer, the annulus permeability, and the screen permeability are initialized. Use formula (2) to calculate the current mechanical sand control skin coefficient. After entering the next time step, use 


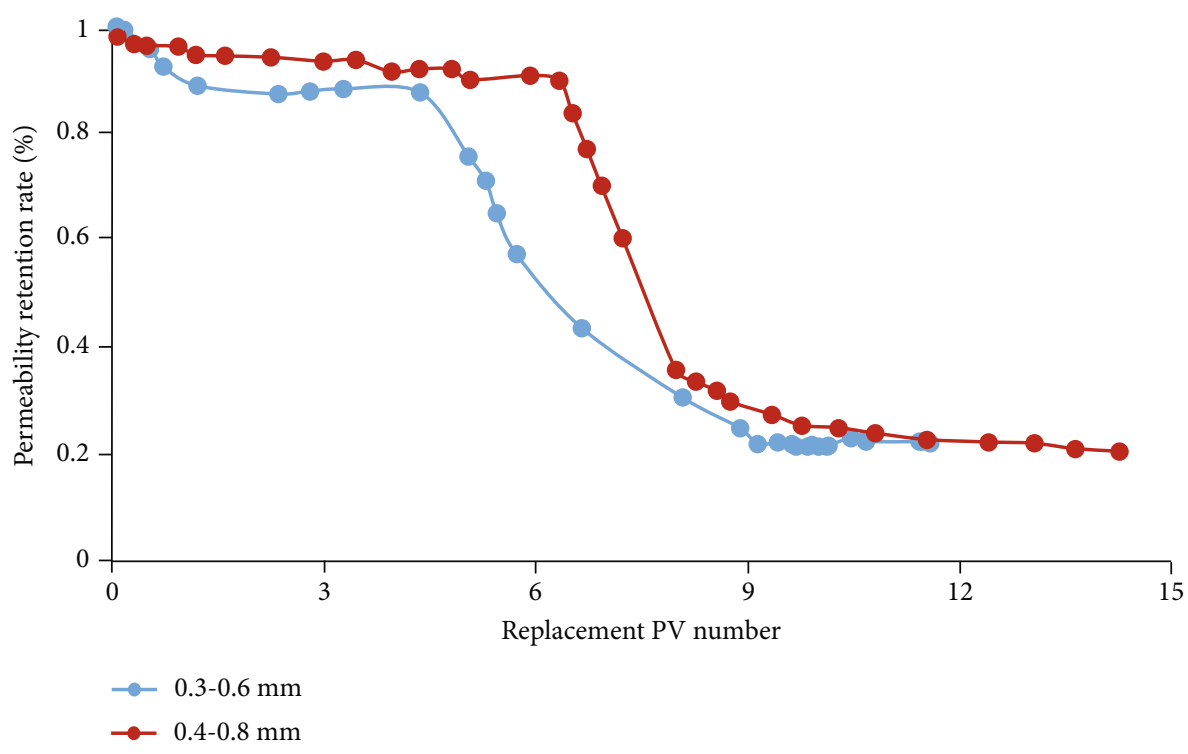

FIGURE 5: Blocking process of sand control system in pipe.

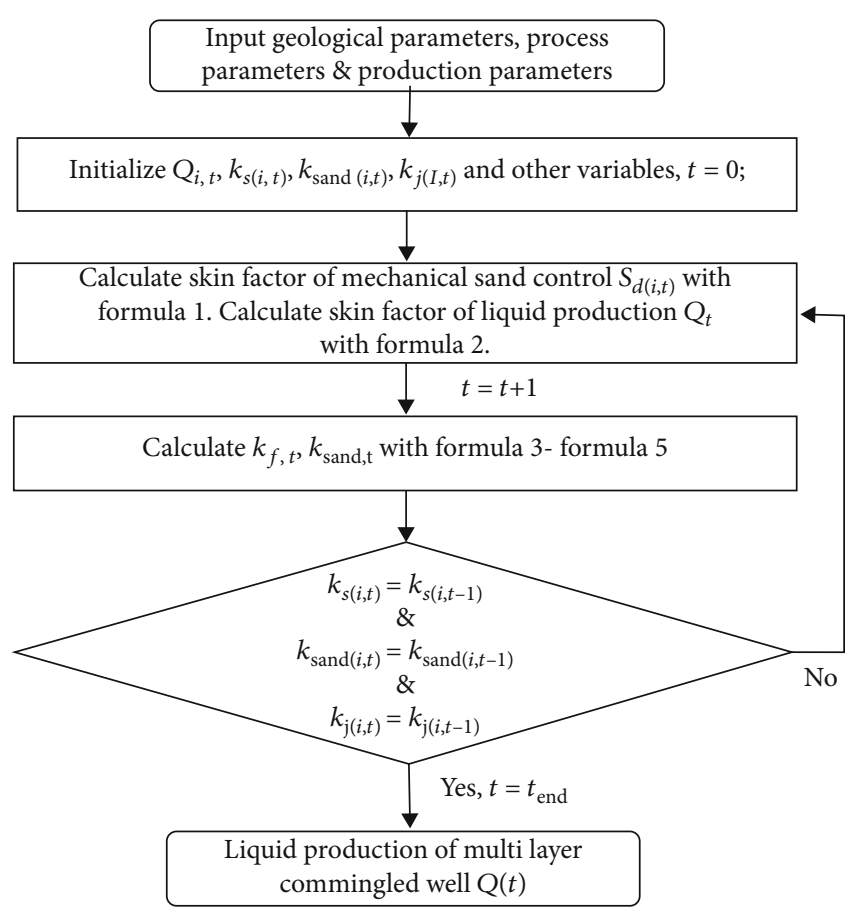

FIGURE 6: Productivity calculation process of multilayer combined production well.

formulas (3), (4), and (5) obtained by indoor experimental fitting to calculate the current squeeze packing permeability, annulus permeability, and screen permeability. When the rate of the above three permeability is not changing, it proves that the production of the oil well has entered a steady state, and the output of the output oil well after the production is reduced to stable dynamic data.

The productivity model can be used to further calculate the impact of different plugging radii and different plugging degrees on the productivity of oil wells. The plugging process extends from far wells to near wells. Analyze the relationship between plugging location and productivity. As shown in Figure 7, the closer the plugging location is to the wellbore, the greater the productivity loss of the oil well, the greater the permeability loss of the gravel pack, and the greater the productivity loss of the oil well. The production process shows a gradual decline in output. As shown in Figure 8, when the casing is filled with gravel and the screen begins to block, the skin coefficient rises sharply, the oil well productivity is less than $20 \%$, and the sand control fails. Therefore, expanding the sand control radius of squeeze packing can block the particles as far as possible in the formation away from the wellbore, which helps protect the productivity of the oil well. It is believed that when the filling radius reaches $5 \mathrm{~m}$, the impact of blockage on productivity is less than $30 \%$. For the target layer of liquid extraction, the filling radius should be enlarged to reduce the influence of blockage on its liquid volume.

3.2. Optimization of Filling Radius of Layered Sand Control Extrusion. Siltstone reservoirs are mostly controlled by fluvial facies, with many vertical intervals and poor horizontal continuity. During the development process, a single well encountered many oil layers, and the physical properties of the combined production intervals varied greatly. For this type of reservoir, in the high water cut period, it is necessary to selectively increase the liquid volume of the relatively low production level and low water cut interval and control the liquid volume of the high water cut interval. The sand control technology for silt sandstone reservoirs mainly optimizes layering parameters, packing radius, screen sand retaining accuracy, and packing particle size. The range of choice for filling particle size and screen sand retaining accuracy of specific reservoirs is not large. Therefore, through theoretical model productivity calculation, the impact of filling radius of each small layer on oil well productivity is analyzed. The parameters are shown in Table 1. In stratified sand control, stratification is carried out according to the standard that the permeability difference is less than 3 . 


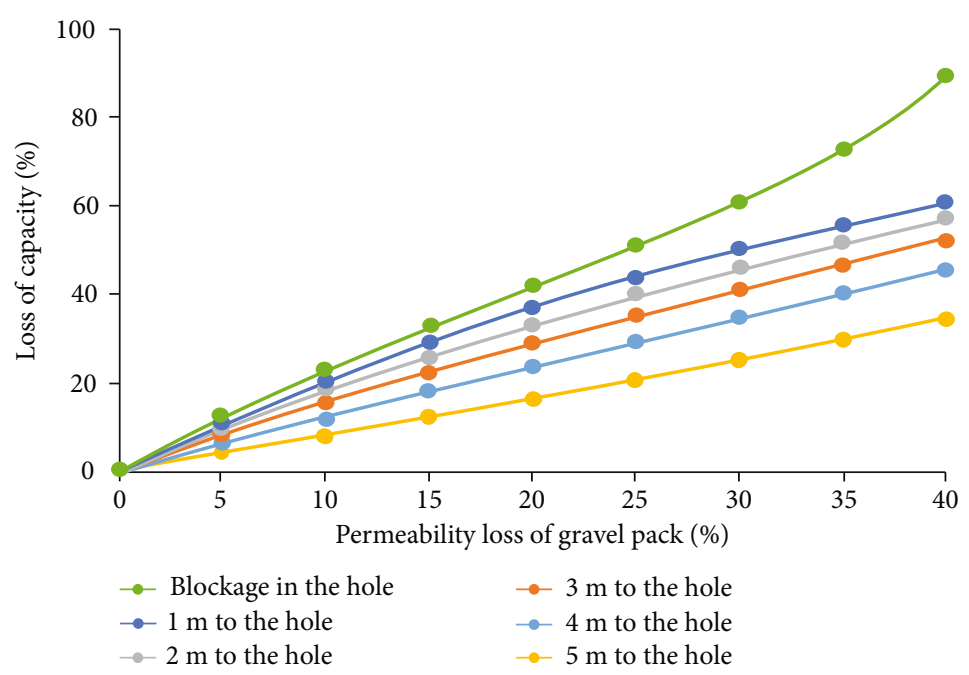

FIGURE 7: The impact of different blockage positions on production capacity.

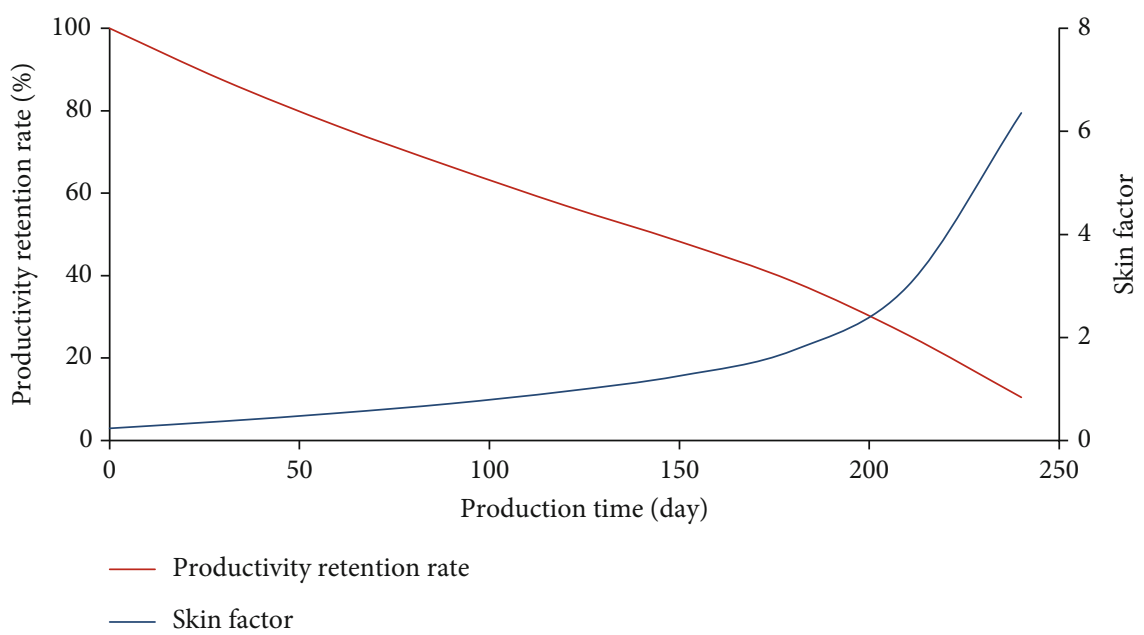

FIGURE 8: Productivity skin coefficient and productivity changes after oil wells are put into production.

For the interval with lower permeability, a larger filling radius is designed, and for the interval with higher permeability, the filling radius is reduced.

As shown in Figure 9, the liquid production of each layer of layered sand control and general sand control under different working systems is compared. The liquid production of the medium- and low-permeability zones of the multilayer combined inclined wells filled with layers is significantly increased, and the high-permeability layer zone fluid production decreases. The stratified packing can increase the liquid volume of the low-medium-permeability layer and control the liquid volume of the high-permeability layer while the overall liquid volume of the oil well is stable. Highpermeability intervals tend to have high water content, where layered filling and sand control can play a role in adjusting the liquid production profile. In siltstone oil reservoirs, the layered sand control technology increases the filling radius in the lower-permeability interval and reduces the filling radius in the higher-permeability interval to improve the interlayer production.

\section{Practice of Sand Control and Liquid Extraction in Kendong \#12 Fault Block}

The Kendong \#12 Sea Oil Production Platform is located in the shallow sea area in the east of Xintan oilfield, most of which are located in the water depth of 0 m-5 m and structurally located in the eastern slope belt of Kendong Uplift. Geological reserves are $1759 \times 10^{4} \mathrm{t}$. After more than 10 years of development, it plans to exit the environmentally sensitive area. The current water content is $86 \%$, and the recovery level is $15 \%$. Before exiting, it will carry out enhanced oil production.

The Minghua Town and Guanshang Section of the target block are deposited by meandering rivers. The meandering sand dam of the main river channel is the main reservoir with a buried depth of $700 \mathrm{~m} \sim 1200 \mathrm{~m}$. The grains are mainly argillaceous cementation, the cementation type is mostly contact type, and the rock type is detrital feldspar sandstone. The average particle size is $0.22 \mathrm{~mm}$, which is a typical silty sandstone reservoir. 
TABLE 1: Main parameters of multilayer combined production capacity calculation.

\begin{tabular}{lccc}
\hline \multicolumn{2}{c}{ Geological parameters } & \multicolumn{2}{c}{ Process parameters } \\
\hline Sand body layers & 7 & Very poor permeability throughout the well & 5.5 \\
Sand body thickness $(\mathrm{m})$ & $5-15$ & Very poor permeability after stratification & $1.5,3$ \\
Number of interlayers & 7 & Sand body layers & 7 \\
Interlayer properties & Impermeable & Hole drift angle ( ${ }^{\circ}$ ) & 30 \\
Porosity (\%) & $25-35$ & Filling particle size (mm) & $0.4-0.8$ \\
Permeability $\left(10^{-3} \mu \mathrm{m}^{2}\right)$ & $1000-5500$ & Shooting layer (m) & $1400-1490$ \\
Pressure coefficient & 1.01 & General filling radius (m) & 2 \\
Rock compressibility $\left(\mathrm{MPa}^{-1}\right)$ & $2 \times 10^{-4}$ & Layered filling radius (m) & Mediate- and low-permeability layer 3; \\
& & & high-permeability layer 1 \\
\hline
\end{tabular}

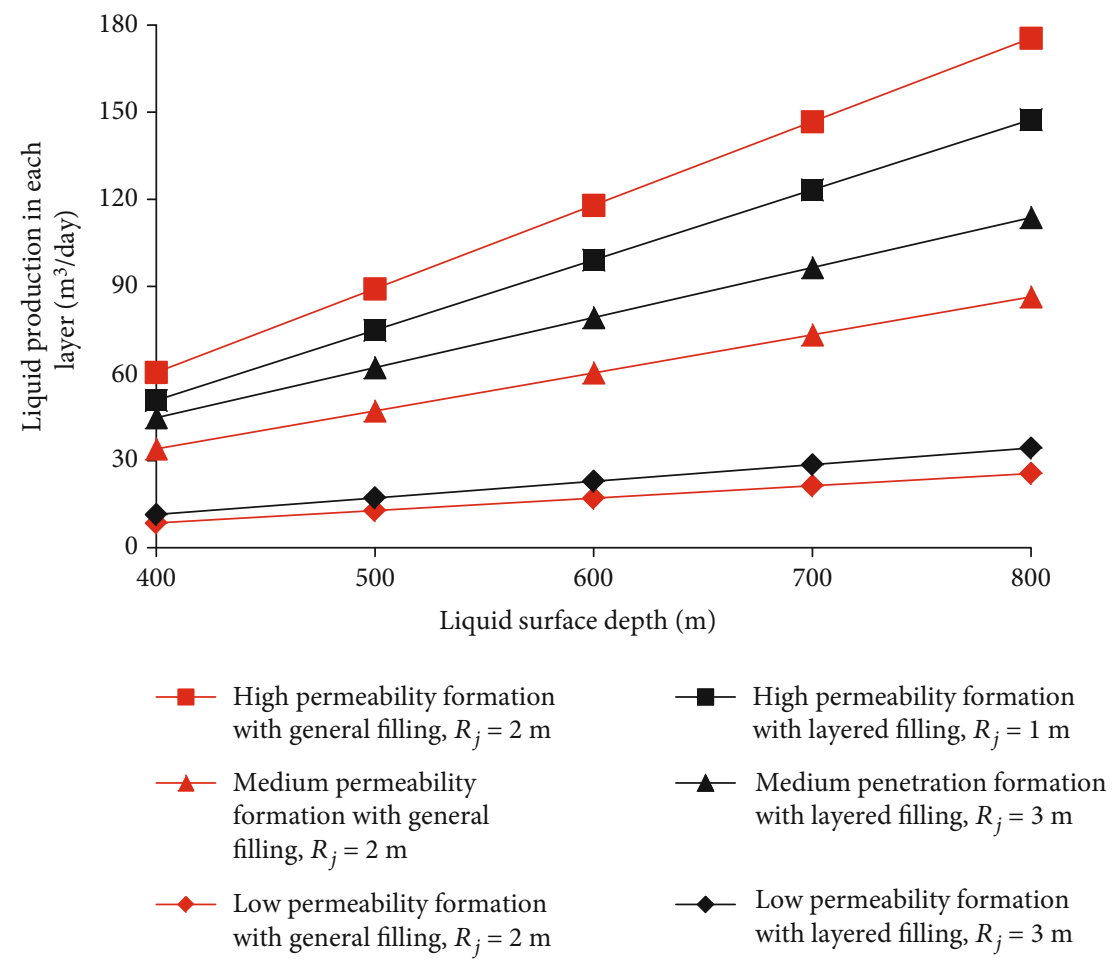

FIgURE 9: The fluid production of each layer in a multilayer combined well with different sand control methods.

The oil-bearing well section of Kendong 12 fault block is $363 \mathrm{~m}$ long in the vertical direction, and the oil layer varies greatly in the horizontal direction and the distribution is unstable. The thickness of the single oil layer is thin, the maximum single layer thickness is 6.7 meters, and the minimum single layer thickness is 0.7 meters. The number of oil layers greater than 3 meters accounted for only $33 \%$, and the number of oil layers less than 3 meters accounted for $67 \%$. In order to increase the drilling encounter rate, the well type of this block is mainly inclined vertical wells with long sections.

The opening conditions of XTKD12-X9 are shown in Table 2. The well has a large oil layer span and large interlayer differences. The Nm layer has high water content since its development, and the water content is $95.02 \%$ during single layer production. Therefore, the Ng6 hole is supplemented, and the upper and lower layers are resand control for combined mining. Through layered sand control productivity analysis, the well is designed for sand control and fluid extraction. The design adopts layered extrusion packing for sand control, with Nm712 and Nm712 as the upper layer for extrusion packing and Ng612 and Ng62 as the lower layer for extrusion packing and sand control. Using the median particle size of $0.65 \mathrm{~mm}$, the permeability of the gravel layer was measured to be $8000 \times 10^{-3} \mu \mathrm{m}^{2}$, and the permeability loss of the testing sand belt was $18 \%$. Using the multilayered production capacity model, a total of 25 sets of digital simulation experiments were enumerated under the condition of a 
TABLE 2: Profile of XTKD12-X9 well intervals.

\begin{tabular}{|c|c|c|c|c|c|c|c|c|}
\hline $\begin{array}{l}\text { Serial } \\
\text { number }\end{array}$ & Level & $\begin{array}{c}\text { Reservoir } \\
\text { interval }(\mathrm{m})\end{array}$ & $\begin{array}{c}\text { Sand layer } \\
\text { thickness (m) }\end{array}$ & $\begin{array}{l}\text { Electrical test } \\
\text { interpretation }\end{array}$ & $\begin{array}{l}\text { Permeability } \\
\left(10^{-3} \mu \mathrm{m}^{2}\right)\end{array}$ & $\begin{array}{c}\text { Porosity } \\
(\%)\end{array}$ & $\begin{array}{l}\text { Actual shot } \\
\text { section }(\mathrm{m})\end{array}$ & $\begin{array}{l}\text { Actual shot } \\
\text { thickness (m) }\end{array}$ \\
\hline 1 & $\mathrm{Nm} 712$ & $1025.9-1029.8$ & 3.9 & Oil layer & 590 & 38.3 & $1025.9-1029.8$ & 3.9 \\
\hline 2 & $\mathrm{Nm} 712$ & $1031.3-1036.3$ & 5 & Oil layer & 373 & 33 & $1031.3-1036.3$ & 5 \\
\hline 3 & $\mathrm{Nm} 721$ & $1046.4-1055.1$ & 8.7 & Oil layer & 494 & 35 & $1046.4-1055.1$ & 8.7 \\
\hline 4 & Ng311 & & & Flooded & & & $1389-1393$ & 4 \\
\hline 5 & Ng312 & & & Flooded & & & $1400.5-1403.5$ & 3 \\
\hline 6 & Ng321 & & & Flooded & & & $14101-418$ & 8 \\
\hline 7 & Ng612 & $1599.3-1606.4$ & 7.1 & Oil layer & 297 & 32.7 & $1600-1606$ & 6 \\
\hline 8 & Ng62 & $1625.1-1635.1$ & 10 & Oil layer & 189 & 30.3 & $1626.1-1635$ & 8.9 \\
\hline
\end{tabular}
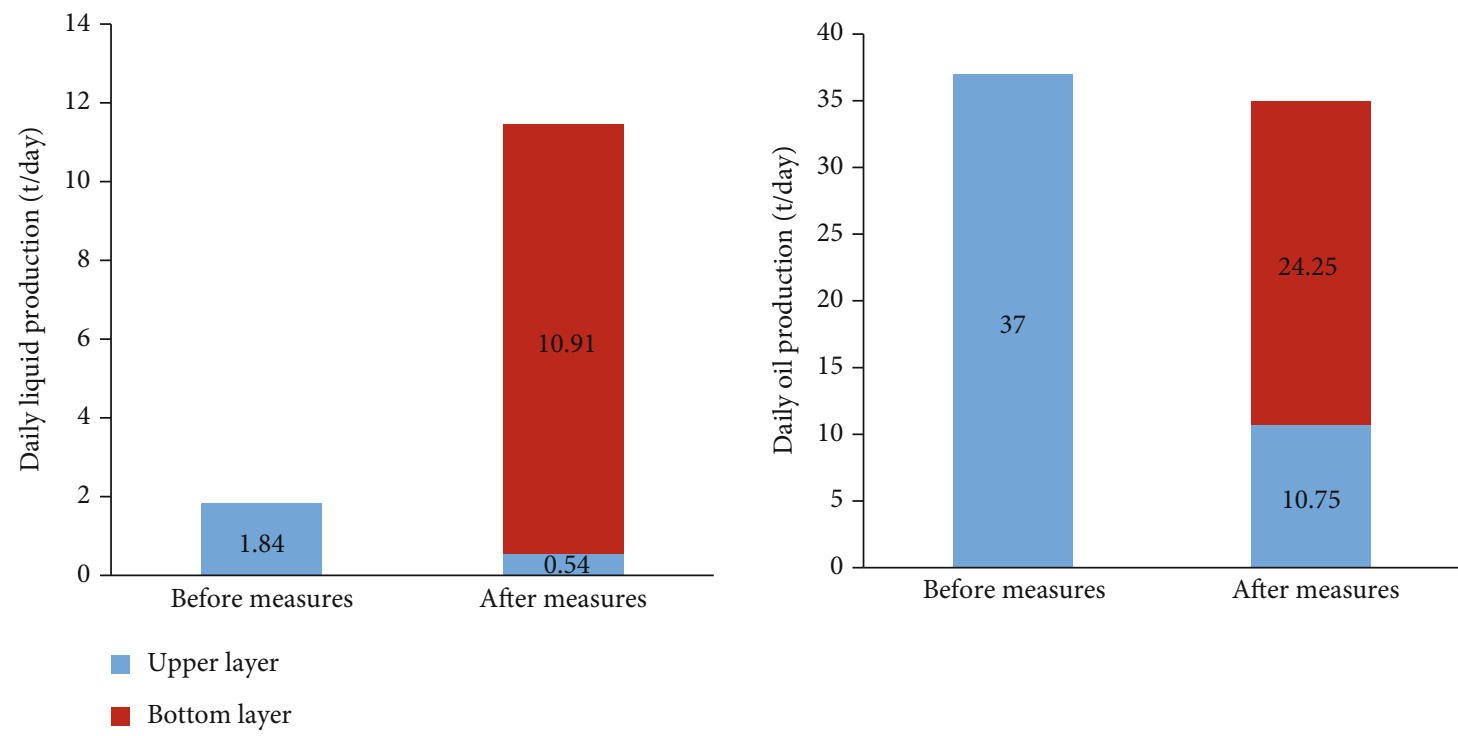

Figure 10: Production comparison of well XTKD12-X9 before and after measures.

filling radius of $1 \mathrm{~m}-5 \mathrm{~m}$. The sand control radius is optimized with the goal of increasing the liquid volume in the lower layer, and the sand control radius parameters are obtained: the upper sand control radius is $2 \mathrm{~m}$, the lower sand control radius is $5 \mathrm{~m}$, and the liquid level is controlled to be lower than 450 meters for production.

After the layered sand control of well XTKD12-X9 was implemented, the production was controlled to be lower than $450 \mathrm{~m}$. The production comparison before and after the measures is shown in Figure 10. At present, the well has 36.7 cubic meters of daily fluid, 12.5 tons of daily oil, and $66 \%$ water cut. The production of each layer is split to show that the layered filling process has played a better role in adjusting the liquid production profile and can improve the production effect of the relatively low-permeability layer.

The Kendong \#12 block widely used multilayer combined production and fluid extraction and sand control technology in the enhanced oil production stage before exiting, optimizing the filling parameters, the nonmain layer fluid production strength was improved, and the production effect was improved. After the measures were implemented, the block oil production rate increased from $1.08 \%$ to $2.12 \%$, and the oil well failure rate remained stable. After the measures, the oil well sand control continued to be effective.

\section{Conclusions}

(1) Laboratory experiments were carried out to analyze the clogging process of the inclined vertical well in siltstone reservoirs. The migration of formation particles is the main cause of blockage. The blockage extends from the packing layer to the wellbore. The closer the blockage is to the wellbore, the greater the permeability loss of the gravel pack and the greater the productivity loss of the oil well

(2) The blockage of the in-pipe sand control system has a great impact on the productivity of oil wells, which can reduce the productivity of oil wells by more than 90\%. Enlarging the squeeze packing sand control radius can block particles away from the wellbore and protect the productivity of oil wells. For the 
target layer of liquid extraction, the filling radius should be enlarged to reduce the influence of blockage on its liquid volume

(3) The layered sand control technique can increase well productivity, increase the filling radius in the layers with lower permeability, and reduce the filling radius in the layers with higher permeability, which can adjust the liquid production profile and expand the longitudinal drainage area. The effect of stratified sand control and liquid extraction in Kendong \#12 siltstone reservoir shows that the stratified filling technology can improve the production effect of low- to mediate-permeability layers, and the oil recovery of the block is significantly improved

\section{Data Availability}

All data do include in the paper.

\section{Conflicts of Interest}

The authors declare that they have no conflicts of interest.

\section{Acknowledgments}

This work is supported by the Major Science and Technology Programs of SINOPEC, Shengli Oilfield 2021 Oil and Gas Production Basic Prospective Project, and Independent Innovation Research Project of China University of Petroleum (East China) with No. 20CX06089A.

\section{References}

[1] Y. Lin, Z. Yu, and Z. Zhenyong, "Experimental study of shale damage by hydration under triaxial stress state," Petroleum Drilling Techniques, vol. 24, no. 1, pp. 22-23, 1996.

[2] Z. Ran, Y. Wei Guo, L. Haipeng, and Z. Wanmao, "Wellbore crustal stress's key factors analysis of shed and reservoir," Science Technology and Engineering, vol. 16, no. 17, pp. 138-144, 2016.

[3] F. Xingwo, L. Xiangfang, and G. Wenlong, "Research on sand production mechanism at home and abroad," Drilling and Production Technology, vol. 27, no. 3, pp. 69-70, 2004.

[4] N. Morita and P. A. Boyd, "Typical sand production problem. Case studies and strategies for sand control," in SPE Annual Technical Conference and Exhibition, Dallas, TX, 1991.

[5] N. Morita, D. L. Whitfill, I. Massie, and T. W. Knudsen, "Realistic sand-production prediction: numerical approach," SPE Production Engineering, vol. 4, no. 1, pp. 15-24, 1989.

[6] N. Morita, D. L. Whitfill, O. P. Fedde, and T. H. Levik, "Parametric study of sand-production prediction: analytical approach," SPE Production Engineering, vol. 4, no. 1, pp. 2533, 1989.

[7] L. C. B. Bianco, Phenomena of Sand Production in NonConsolidated Sandstone, [Ph.D. thesis], The Pennsylvania State University, State College, PA, 1999.

[8] C. D. Hall Jr. and W. H. Harrisberger, "Stability of sand arches: a key to sand control," Journal of Petroleum Technology, vol. 22, no. 7, pp. 821-829, 1970.
[9] H. Hongwei and C. Ping, "Research on micro-mechanism of softening and argillitization of mudstone," Journal of Tongji University (Natural Scinece), vol. 35, no. 7, pp. 865-870, 2007.

[10] B. Liang, X. Y. Tan, L. G. Jiang, and B. B. Jiao, "Expevimental analysis of slaking characteristics of mudstone in different PH solutions," Journal of Civil, Arvhitecturalङ Environmental Engineering., vol. 37, no. 2, pp. 23-28, 2015.

[11] L. Yuguo, J. Peifeng, C. Gang, Z. Yizhong, Z. Yucheng, and S. Yingfei, "Uniform acidizing technology for horizontal wells in unconsolidated sandstone reservoirs," Fault-Block Oil \& Gas Field, vol. 26, no. 6, pp. 805-809, 2019.

[12] Z. Wu, C. Cui, G. Lv, S. Bing, and G. Cao, "A multi-linear transient pressure model for multistage fractured horizontal well in tight oil reservoirs with considering threshold pressure gradient and stress sensitivity," Journal of Petroleum Science and Engneering, vol. 172, pp. 839-854, 2019.

[13] Z. Wu, L. Dong, C. Chuanzhi, X. Cheng, and Z. Wang, "A numerical model for fractured horizontal well and production characteristics: comprehensive consideration of the fracturing fluid injection and flowback," Journal of Petroleum Science and Engneering, vol. 187, p. 106765, 2020.

[14] S. Wang, F. Lai, K. Wang, Z. Li, and H. Wang, "Development favorable area and productivity potential evaluation method of a tight oil reservoir," Geofluids, vol. 2021, 14 pages, 2021.

[15] Z. Yuan, L. Yang, Y. Zhang et al., "Productivity evaluation for long horizontal well test in deep-water faulted sandstone reservoir," Geofluids, vol. 2020, Article ID 8845270, 10 pages, 2020.

[16] M. du, X. Yao, S. Zhang, H. Zhou, C. Wu, and J. Zhao, "Geochemical characteristics and productivity response of produced water from coalbed methane wells in the Yuwang block, eastern Yunnan, China," Geofluids, vol. 2020, Article ID 5702031, 11 pages, 2020.

[17] T. K. Perkins and J. S. Weingarten, "Stability and failure of spherical cavities in unconsolidated sand and weekly consolidated rock," in SPE Annual Technical Conference and Exhibition. Society of Petroleum Engineers, Houston, TX, 1988.

[18] L. C. B. Bianco and P. M. Halleck, "Mechanisms of arch instability and sand production in two-phase saturated poorly consolidated sandstones," in SPE European Formation Damage Conference. Society of Petroleum Engineers, The Hague, The Netherlands, 2001.

[19] X. Youming and Y. Pan, "Study on productivity predict ion of the horizontal wels with completion methods of perforation series," Journal of Southwest Petroleum University, vol. 18, no. 2, pp. 57-62, 1996.

[20] L. Huiqing, Advanced Reservoir Engineering, Petroleum Industry Press, Beijing, 2016.

[21] C. Dong, Oil well sand control theory and technology, China Petroleum University Press, Dongying, 2012. 